\title{
A Note on Finding Geodesic Equation of Two Parameters Gamma Distribution
}

\author{
William W. S. Chen \\ Department of Statistics, The George Washington University, Washington DC, USA \\ Email: Williamwschen@gmail.com
}

Received 23 September 2014; revised 20 October 2014; accepted 11 November 2014

Copyright (C) 2014 by author and Scientific Research Publishing Inc.

This work is licensed under the Creative Commons Attribution International License (CC BY). http://creativecommons.org/licenses/by/4.0/

c) (i) Open Access

\begin{abstract}
Engineers commonly use the gamma distribution to describe the life span or metal fatigue of a manufactured item. In this paper, we focus on finding a geodesic equation of the two parameters gamma distribution. To find this equation, we applied both the well-known Darboux Theorem and a pair of differential equations taken from Struik [1]. The solution proposed in this note could be used as a general solution of the geodesic equation of gamma distribution. It would be interesting if we compare our results with Lauritzen's [2].
\end{abstract}

\section{Keywords}

Darboux Theorem, Geodesic Equation, Differential Equation, Gamma Distribution

\section{Introduction}

Rao [3] introduced a Riemannian metric over the space of a parametric family of probability distribution. He proposed the minimized distance induced by the metric as a measure of dissimilarity between probability distribution. In recent year, there has been an increasing interest in the study of geometrical properties. For example, Lauritzen derived the Gaussian Manifold, Inverse Gaussian Manifold and Geodesic Equation of Gamma Manifold. In the Gamma Case, he found no general explicit solution for geodesic equations, except in the special case when $\alpha=1$. Mitchell [4] worked on statistical manifolds of univariate or multivariate elliptic distributions and found the $\alpha$ Gaussian Curvature and geodesics for the univariate elliptic class. Oller [5] provided the Gaussian Curvature and Rao Distance of extreme value probability distributions such as Gumbel, Cauchy-Frechet, Weibull and the Logistic Probability Distribution. Chen [6] presents a comparison of curvature between Gaussian or Riemann. Chen and Kotz [7] have studied the Riemannian structure of the three-parameter gamma distribution. In this note, we will focus on gamma distributions. We define the elements of the Fisher Information Matrix as the Coefficients of the First Fundamental Form. Then we will apply the existing theorems or proper- 
ties in classical differential geometry to find the geodesic equation of gamma distribution. Applying these results with the Darboux Theory [8] helps us find a natural solution of the Geodesic Equation. Alternatively, we will also apply the traditional technique of finding the Geodesic Equation of the Gamma Manifold in order to compare it with the Darboux Approach. As expected, the two results are identical.

\section{The Geodesic Equation}

In general, we can use standard notation to represent the distance between two points $P$ and $Q$ on a curve,

$$
\mathrm{d} s^{2}=E \mathrm{~d} v^{2}+2 F \mathrm{~d} u \mathrm{~d} v+G \mathrm{~d} u^{2}
$$

However, if we can transform the distance function (2.1) to the following simplified form

$$
\mathrm{d} s^{2}=\mathrm{d} z^{2}+\sigma^{2} \mathrm{dz} z_{1}^{2}
$$

it could help us to find the Geodesic Equation more easily. The task of transforming Equation (2.2) is equivalent to asking how we can determine two independent functions, $z=z(v, u)$, and $z_{1}=z_{1}(v, u)$, such that Equation (2.1) can be transformed into Equation (2.2). Since $z(v, u)$ is a function of $(u, v)$, we know from calculus that

$$
\mathrm{d} z=z_{v} \mathrm{~d} v+z_{u} \mathrm{~d} u \text {, thus, } \mathrm{d} s^{2}-\mathrm{d} z^{2}=\left(E-z_{v}^{2}\right) \mathrm{d} v^{2}+2\left(F-z_{u} z_{v}\right) \mathrm{d} u \mathrm{~d} v+\left(G-z_{u}^{2}\right) \mathrm{d} u^{2}
$$

If we assume that (2.2) is valid, then it would be necessary for either the right hand side of (2.3) to be a perfect square, or for the determinant of (2.3) to be equal to zero. That is,

$$
\left(F-z_{u} z_{v}\right)^{2}-\left(E-z_{v}^{2}\right)\left(G-z_{u}^{2}\right)=0
$$

Equation (2.4) can be rewritten as

$$
\frac{E z_{u}^{2}-2 F z_{u} z_{v}+G z_{v}^{2}}{E G-F^{2}}=1
$$

for convenience, we usually write the left-hand side of (2.5) as $\nabla Z=1$.

Now, if we wish to find a general solution to (2.5), then we should rewrite (2.3) in the following form:

$$
\mathrm{d} s^{2}-\mathrm{d} z^{2}=(m(v, u) \mathrm{d} v+n(v, u) \mathrm{d} u)^{2}
$$

where both $m, n$ are some known function of $u$ and $v$.

Furthermore, if we can find an integration factor $\frac{1}{\sigma}$ such that, $m(v, u) \mathrm{d} v+n(v, u) \mathrm{d} u=\sigma \mathrm{d} z_{1}$, then the distance function $\mathrm{ds}^{2}$ could be transformed into the form (2.2). Summarizing the above procedures, we conclude that in order to find the geodesic equation, two steps must be completed: Step 1: we must find a general solution of the partial differential Equation (2.5); Step 2: we must find an integration factor of Equation (2.6). Darboux proposed an improved method to combine the two steps into one step; that method is stated in the following theorem.

Theorem 1: Assume the given partial differential equation $\nabla Z=1$ has an arbitrary solution $Z=Z(u, v, a)$, where a is an arbitrary constant. Then $\frac{\partial Z(u, v ; a)}{\partial a}=$ constant is the required geodesic equation.

Proof: See reference [8].

Form Section 3, we know that the coefficient of the first fundamental form is given as:

$$
E=\frac{u}{v^{2}}, F=0, G=\psi^{\prime}(u)-\frac{1}{u} \text { or } \frac{\frac{u}{v^{2}} z_{u}^{2}+\left(\psi^{\prime}(u)-\frac{1}{u}\right) z_{v}^{2}}{\left(\psi^{\prime}(u)-\frac{1}{u}\right) \frac{u}{v^{2}}}=1
$$

To solve the partial differential equation above, we adopt the separate variable method and 


$$
v^{2} z_{v}^{2}=\frac{u \psi^{\prime}(u)-1-u z_{u}^{2}}{\psi^{\prime}(u)-\frac{1}{u}}=A^{2}
$$

hence, form the relation $v^{2} z_{v}^{2}=A^{2}$, we have $v z_{v}=A$ or $Z=A \ln v$.

$$
\begin{aligned}
& \text { Also, form the relation } \frac{u \psi^{\prime}(u)-1-u Z_{u}^{2}}{\psi^{\prime}(u)-\frac{1}{u}}=A^{2} \text {, we could solve } \\
& \qquad Z_{u}^{2}=\left(\psi^{\prime}(u)-\frac{1}{u}\right)\left(1-\frac{A^{2}}{u}\right), Z= \pm \sqrt{\left(\psi^{\prime}(u)-\frac{1}{u}\right)\left(1-\frac{A^{2}}{u}\right)} \mathrm{d} u
\end{aligned}
$$

We find one of the general solutions:

$$
Z=A \ln v \pm \int \sqrt{\left(\psi^{\prime}(u)-\frac{1}{u}\right)\left(1-\frac{A^{2}}{u}\right)} \mathrm{d} u
$$

Thus, by applying the Darboux Theorem, we can find the geodesic equation of the gamma distribution

$$
\frac{\partial Z}{\partial A}=\ln v \pm \int \frac{A}{u} \sqrt{\frac{u \psi^{\prime}(u)-1}{u-A^{2}}} \mathrm{~d} u=B, \ln v \pm \int \frac{A}{u} \sqrt{\frac{u \psi^{\prime}(u)-1}{u-A^{2}}} \mathrm{~d} u=B
$$

where $A, B$ are arbitrary constants.

Another method to find the geodesic equation of the gamma distribution is by solving a pair of differential equations given in the Appendix. When $\alpha=+1$, we called it the exponential connection where $u$ is a straight line in the $(v, u)$-plane. When $\alpha=-1$, we called it the mixture connection where $v$ is a straight line in the $(v, u)$-plane. When $\alpha=0$, the Riemannian connection is the most important one and we seek its solution in the following section.

$$
\begin{gathered}
\frac{\mathrm{d}^{2} v}{\mathrm{~d} s^{2}}-\frac{1}{v}\left(\frac{\mathrm{d} v}{\mathrm{~d} s}\right)^{2}+\frac{1}{u} \frac{\mathrm{d} u \mathrm{~d} v}{\mathrm{~d} s \mathrm{~d} s}=0 \\
\frac{\mathrm{d}^{2} u}{\mathrm{~d}^{2} s}-\frac{u}{2 v^{2}\left(u \psi^{\prime}(u)-1\right)}\left(\frac{\mathrm{d} v}{\mathrm{~d} s}\right)^{2}+\frac{u^{2} \psi^{\prime \prime}(u)+1}{2 u\left(u \psi^{\prime}(u)-1\right)}\left(\frac{\mathrm{d} u}{\mathrm{~d} s}\right)^{2}=0
\end{gathered}
$$

and the distance function

$$
\mathrm{ds}^{2}=\frac{u}{v^{2}} \mathrm{~d} v^{2}+\left(\psi^{\prime}(u)-\frac{1}{u}\right) \mathrm{d} u^{2}
$$

We need only two out of three of the above equations to find our Gamma Geodesic Equation.

We will choose the first and third equations. To simplify the notation, we let

$$
p=\frac{\mathrm{d} v}{\mathrm{~d} s}, \frac{\mathrm{d} p}{\mathrm{~d} s}=\frac{\mathrm{d}^{2} v}{\mathrm{ds} s^{2}}
$$

So the first equation becomes: $\frac{\mathrm{d} p}{\mathrm{~d} s}+\frac{1}{u} p \frac{\mathrm{d} v}{\mathrm{~d} s}-\frac{1}{v} p^{2}=0$.

Dividing this equation by factor $p$, we get: $\ln p+\ln u-\ln v=\ln C=A$, or $\frac{\mathrm{d} v}{\mathrm{~d} s}=A \frac{v}{u}$, where $C$ and $A$ are arbitrary constants.

Thus, $\mathrm{d} s=\frac{u \mathrm{~d} v}{A v}$ and, associating with the third equation, we derive the following separate variables equation:

$$
\frac{\mathrm{d} v}{v}= \pm \frac{A}{u} \sqrt{\frac{\left(u \psi^{\prime}(u)-1\right)}{u-A^{2}} \mathrm{~d} u}
$$


Integrating on both sides, we finally get:

$$
\ln v \pm \int \frac{A}{u} \sqrt{\frac{\left(u \psi^{\prime}(u)-1\right)}{u-A^{2}} \mathrm{~d} u}=B
$$

where $A$ and $B$ are arbitrary constants.

\section{List the Fundamental Tensor}

The probability density function for the gamma distribution is given by

$$
f(x ; v, u)=\frac{\left(\frac{u}{v}\right)^{u} x^{u-1}}{\Gamma(u)} \mathrm{e}^{-\frac{x u}{v}}, 0<x<\infty, u>0, v>0
$$

where $u$ and $v$ are parameters

$$
\ln f=u(\ln u-\ln v)+(u-1) \ln x-\ln \Gamma(u)-\frac{x u}{v}
$$

$f(v, u)$ are two parameters of the probability density functions. From Equation (3.1), we derive $(v, u)$-parametrization of the fundamental metric tensor components, or the Fisher Information Matrix.

$$
E=-E\left(\frac{\partial^{2} \ln f}{\partial v^{2}}\right)=\frac{u}{v^{2}}, F=-E\left(\frac{\partial^{2} \ln f}{\partial v \partial u}\right)=0, G=-E\left(\frac{\partial^{2} \ln f}{\partial u^{2}}\right)=\psi^{\prime}(u)-\frac{1}{u}
$$

where $\psi(u)=\frac{\mathrm{d}}{\mathrm{d} u} \ln \Gamma(u)$ is the digamma function and $\psi^{\prime}(u), \psi^{\prime \prime}(u)$ are the polygamma functions.

It is common to use tensor notation to $E, F$ and $G$, i.e. $E=g_{11}, F=g_{12}$ and $G=g_{22}$.

It is clear that $E, F$ and $G$ are functions of the parameters $v$ and $u$. The expectations apply to the sample space where the random variables are defined. Then the matrix and its inverse matrix can be given as follows:

$$
g=\left(\begin{array}{cc}
\frac{u}{v^{2}} & 0 \\
0 & \psi^{\prime}(u)-\frac{1}{u}
\end{array}\right), g^{-1}=\left(\begin{array}{cc}
\frac{v^{2}}{u} & 0 \\
0 & \frac{u}{u^{*} \psi^{\prime}(u)-1}
\end{array}\right)
$$

This section lists the Christoffel Symbols of the first kind of Riemannian connection of distribution (3.1).

$$
[11,1]=\frac{-u}{v^{3}},[11,2]=\frac{-1}{2 v^{2}},[21,1]=[12,1]=\frac{1}{2 v^{2}},[12,2]=[21,2]=[22,1]=0,[22,2]=\frac{\psi^{\prime \prime}(u) u^{2}+1}{2 u^{2}}
$$

Following Amari [9] with the $\alpha$-connections, we define a one-parameter family of affine connections when $\alpha=1$

$$
{ }^{1} \Gamma_{111}=\frac{-2 u}{v^{3}},{ }^{1} \Gamma_{121}={ }^{1} \Gamma_{211} \frac{1}{v^{2}},{ }^{1} \Gamma_{212}={ }^{1} \Gamma_{122}={ }^{1} \Gamma_{221}={ }^{1} \Gamma_{112}={ }^{1} \Gamma_{222}=0
$$

The skewness tensors can then be calculated by using the relation from Appendix, i.e. $T_{i j k}=2\left([i j, k]-{ }^{1} \Gamma_{i j k}\right)$

$$
T_{111}=\frac{2 u}{v^{3}}, T_{112}=T_{121}=T_{211}=\frac{-1}{v^{2}}, T_{221}=T_{122}=T_{212}=0, T_{222}=\frac{\psi^{\prime \prime}(u) u^{2}+1}{u^{2}}
$$

Whereby the $\alpha$-connections can be determined as follows:

$$
\begin{aligned}
& { }^{\alpha} \Gamma_{111}=\frac{-(\alpha+1) u}{v^{3}},{ }^{\alpha} \Gamma_{112}=\frac{\alpha-1}{2 v^{2}},{ }^{\alpha} \Gamma_{121}={ }^{\alpha} \Gamma_{211}=\frac{\alpha+1}{2 v^{2}}, \\
& { }^{\alpha} \Gamma_{122}={ }^{\alpha} \Gamma_{212}={ }^{\alpha} \Gamma_{221}=0,{ }^{\alpha} \Gamma_{222}=\frac{-(\alpha-1)\left(\psi^{\prime \prime}(u) u^{2}+1\right)}{2 u^{2}}
\end{aligned}
$$


If we denote $g^{m k}(\theta)$ as the $(m, k)$ entry of the inverse of the information matrix, we can then define the Christoffel symbols of the second kind:

$$
\begin{aligned}
& { }^{\alpha} \Gamma_{11}^{1}=\frac{-(\alpha+1)}{v},{ }^{\alpha} \Gamma_{11}^{2}=\frac{(\alpha-1) * u}{2 v^{2}\left(\psi^{\prime}(u) * u-1\right)},{ }^{\alpha} \Gamma_{12}^{1}={ }^{\alpha} \Gamma_{21}^{1}=\frac{\alpha+1}{2 u}, \\
& { }^{\alpha} \Gamma_{12}^{2}={ }^{\alpha} \Gamma_{21}^{2}={ }^{\alpha} \Gamma_{22}^{1}=0,{ }^{\alpha} \Gamma_{22}^{2}=\frac{-(\alpha-1)\left(\psi^{\prime \prime}(u) * u^{2}+1\right)}{2 u\left(\psi^{\prime}(u) * u-1\right)}
\end{aligned}
$$

The covariant Riemann curvature tensor, a covariant tensor of fourth order, and its Gaussian curvature can be determined as follows:

$$
R_{1212}=\frac{(\alpha+1)(\alpha-1)\left(u * \psi^{\prime \prime}(u)+\psi^{\prime}(u)\right)}{4 v^{2} *\left(u * \psi^{\prime}(u)-1\right)}, K_{12}=\frac{-(\alpha+1)(\alpha-1)\left(u * \psi^{\prime \prime}(u)+\psi^{\prime}(u)\right)}{4 *\left(u * \psi^{\prime}(u)-1\right)^{2}}
$$

Under the assumption of distribution (3.1), we list some useful moments that may help us to derive the above tensors:

$$
\begin{aligned}
& E(x)=v, \operatorname{Var}(x)=E(x-v)^{2}=\frac{v^{2}}{u}, E\left(x^{2}\right)=v^{2}\left(1+\frac{1}{u}\right) \\
& E(\ln x)=\ln \frac{v}{u}+\psi(u), E(x * \ln x)=v\left(\ln \frac{v}{u}+\psi(u+1)\right)
\end{aligned}
$$

\section{References}

[1] Struik, D.J. (1961) Lectures on Classical Differential Geometry. 2nd Edition, Dover Publications, Inc., New York.

[2] Lauritzen, S.L. (1987) Chapter 4: Statistical Manifolds. Differential Geometry in Statistical Inference. Vol. 10, Institute of Mathematical Statistics, Lecture Notes Monograph Series, Hayward, 163-216.

[3] Rao, C.R. (1945) Information and the Accuracy Attainable in the Estimation of Statistical Parameters. Bulletin of Calcutta Mathematical Society, 37, 81-91.

[4] Mitchell, A.F.S. (1988) Statistical Manifolds of Univariate Elliptic Distributions. International Statistical Review, 56, 1-16. http://dx.doi.org/10.2307/1403358

[5] Oller, J.M. (1987) Information Metric for Extreme Value and Logistic Probability Distributions. Sankhya A, 49, 17-23.

[6] Chen, W.W.S. (1998) Curvature: Gaussian or Riemann. International Conference (IISA), McMaster University, Hamilton, October.

[7] Chen, W.W.S. and Kotz, S. (2013) The Riemannian Structure of the Three-Parameter Gamma Distribution. Applied Mathematics, 4, 514-522.

[8] Darboux, G. (1889-1997) Lecons sur la theorie generale des surfaces. Gauthier-Villars, Paris.

[9] Amari, S.I. (1982) Differential Geometry of Curved Exponential Families Curvature and Information Loss. Annals of Statistics, 10, 357-385. http://dx.doi.org/10.1214/aos/1176345779

[10] Gray, A. (1993) Modern Differential Geometry of Curves and Surfaces. CRC Press, Inc., Boca Raton. 


\section{Appendix}

The definition of the Christoffel Symbols of the first kind in terms of the first partial derivatives of the components of the Riemannian metric tensor:

$$
[i j, k]=\frac{1}{2}\left[\frac{\partial g_{i k}}{\partial \theta_{j}}+\frac{\partial g_{j k}}{\partial \theta_{i}}-\frac{\partial g_{i j}}{\partial \theta_{k}}\right]
$$

Amari [9] defined a one-parameter family of affine connections by the $\alpha$-connections with coefficients:

$$
\begin{aligned}
{ }^{\alpha} \Gamma_{i j k}(\theta) & =E\left[\frac{\partial^{2} \ln f(x \mid \theta)}{\partial \theta_{i} \partial \theta_{j}} * \frac{\partial \ln f(x \mid \theta)}{\partial \theta_{k}}\right]+\frac{1}{2}(1-\alpha) E\left[\frac{\partial \ln f(x \mid \theta)}{\partial \theta_{i}} \frac{\partial \ln f(x \mid \theta)}{\partial \theta_{j}} \frac{\partial \ln f(x \mid \theta)}{\partial \theta_{k}}\right] \\
& =[i j, k]-\frac{1}{2} \alpha T_{i j k}
\end{aligned}
$$

where $[i j, k]$ is defined as before and

$$
T_{i j k}=E\left[\frac{\partial \ln f(x \mid \theta)}{\partial \theta_{i}} \frac{\partial \ln f(x \mid \theta)}{\partial \theta_{j}} \frac{\partial \ln f(x \mid \theta)}{\partial \theta_{k}}\right]
$$

with the skewness tensor $(i, j, k=1,2, \cdots, r)$.

The affine connections can also be used to describe the Christoffel symbols of the second kind, ${ }^{\alpha} \Gamma_{i j}^{k}(\theta)$ where for $i, j, k=1,2, \cdots r$

$$
{ }^{\alpha} \Gamma_{i j}^{k}(\theta)={ }^{\alpha} \Gamma_{i j m}(\theta) * g^{m k} \text {, sum on } m
$$

where $g^{m k}$ denotes the entry of the $(m, k)$ th inverse of the inform ation matrix.

Next, we define the six well known Christoffel symbols (see Struik [1], p. 107, Equations (2)-(7) or Gray, A. [10] p. 398) when $\alpha=0$ :

$$
\begin{aligned}
& \Gamma_{11}^{1}=\frac{G E_{v}-2 F F_{v}+F E_{u}}{2\left(E G-F^{2}\right)}, \Gamma_{12}^{2}=\frac{E G_{v}-F E_{u}}{2\left(E G-F^{2}\right)}, \Gamma_{11}^{2}=\frac{2 E F_{v}-E E_{u}-F E_{v}}{2\left(E G-F^{2}\right)}, \\
& \Gamma_{22}^{1}=\frac{2 G F_{u}-G G_{v}-F G_{u}}{2\left(E G-F^{2}\right)}, \quad \Gamma_{12}^{1}=\frac{G E_{u}-F G_{v}}{2\left(E G-F^{2}\right)}, \quad \Gamma_{22}^{2}=\frac{E G_{u}-2 F F_{u}+F G_{v}}{2\left(E G-F^{2}\right)}
\end{aligned}
$$

In case of gamma distribution and $\alpha=0$, we have the following results

$$
\begin{aligned}
& E=\frac{u}{v^{2}}, F=0, G=\psi^{\prime}(u)-\frac{1}{u}, E_{u}=v^{-2}, E_{v}=-2 u v^{-3}, \\
& G_{u}=\psi^{\prime \prime}(u)+u^{-2}=\frac{u^{2} \psi^{\prime \prime}(u)+1}{u^{2}}, G_{v}=0
\end{aligned}
$$

The history of geodesic lines begins with John Bernoulli's solution of the problem of the shortest distance between two points on a convex surface (1697-1698). In this note, our solution for the geodesic equation of gamma distribution depends on a pair of differential equations.

$$
\begin{aligned}
& \frac{\mathrm{d}^{2} v}{\mathrm{~d} s^{2}}+{ }^{\alpha} \Gamma_{11}^{1}\left(\frac{\mathrm{d} v}{\mathrm{~d} s}\right)^{2}+2^{\alpha} \Gamma_{12}^{1}\left(\frac{\mathrm{d} u}{\mathrm{~d} s} \frac{\mathrm{d} v}{\mathrm{~d} s}\right)+{ }^{\alpha} \Gamma_{22}^{1}\left(\frac{\mathrm{d} u}{\mathrm{~d} s}\right)^{2}=0, \\
& \frac{\mathrm{d}^{2} u}{\mathrm{~d} s^{2}}+{ }^{\alpha} \Gamma_{11}^{2}\left(\frac{\mathrm{d} v}{\mathrm{~d} s}\right)^{2}+2^{\alpha} \Gamma_{12}^{2}\left(\frac{\mathrm{d} u}{\mathrm{~d} s} \frac{\mathrm{d} v}{\mathrm{~d} s}\right)+{ }^{\alpha} \Gamma_{22}^{2}\left(\frac{\mathrm{d} u}{\mathrm{~d} s}\right)^{2}=0
\end{aligned}
$$

If we substitute the results of (3.7) into above equations, we obtain the following two equations:

$$
\frac{\mathrm{d}^{2} v}{\mathrm{ds}^{2}}-\frac{\alpha+1}{v}\left(\frac{\mathrm{d} v}{\mathrm{~d} s}\right)^{2}+\frac{\alpha+1}{u}\left(\frac{\mathrm{d} u}{\mathrm{~d} s} \frac{\mathrm{d} v}{\mathrm{~d} s}\right)=0
$$




$$
\frac{\mathrm{d}^{2} u}{\mathrm{~d} s^{2}}+\frac{(\alpha-1) * u}{2 v^{2}\left(u * \psi^{\prime}(u)-1\right)}\left(\frac{\mathrm{d} v}{\mathrm{~d} s}\right)^{2}-\frac{(\alpha-1) *\left(u^{2} * \psi^{\prime \prime}(u)+1\right)}{2 u\left(u * \psi^{\prime}(u)-1\right)}\left(\frac{\mathrm{d} u}{\mathrm{~d} s}\right)^{2}=0
$$

By introducing the Riemann symbols of the first and second kind, respectively.

$$
\begin{gathered}
R_{i j k}^{l}=\frac{\partial}{\partial u_{j}} \Gamma_{i k}^{l}-\frac{\partial}{\partial u_{i}} \Gamma_{j k}^{l}+\Gamma_{i k}^{m} \Gamma_{m j}^{l}-\Gamma_{j k}^{m} \Gamma_{m i}^{l}(\text { sum on } m) \\
R_{i j k l}=R_{i j k}^{m} g_{m l}
\end{gathered}
$$

The Gaussian curvature $K$ can be written:

$$
K=\frac{R_{1212}}{g_{11} g_{22}-g_{12} g_{21}}=\frac{R_{1212}}{E G-F^{2}}
$$


Scientific Research Publishing (SCIRP) is one of the largest Open Access journal publishers. It is currently publishing more than 200 open access, online, peer-reviewed journals covering a wide range of academic disciplines. SCIRP serves the worldwide academic communities and contributes to the progress and application of science with its publication.

Other selected journals from SCIRP are listed as below. Submit your manuscript to us via either submit@scirp.org or Online Submission Portal.
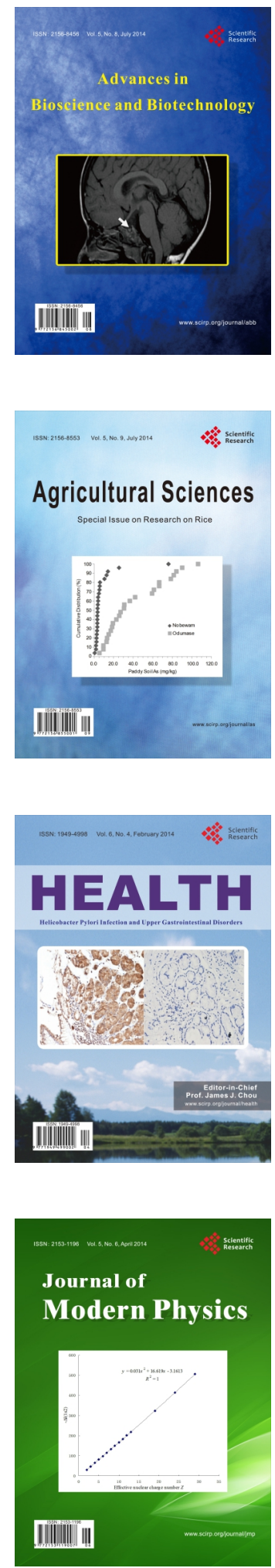
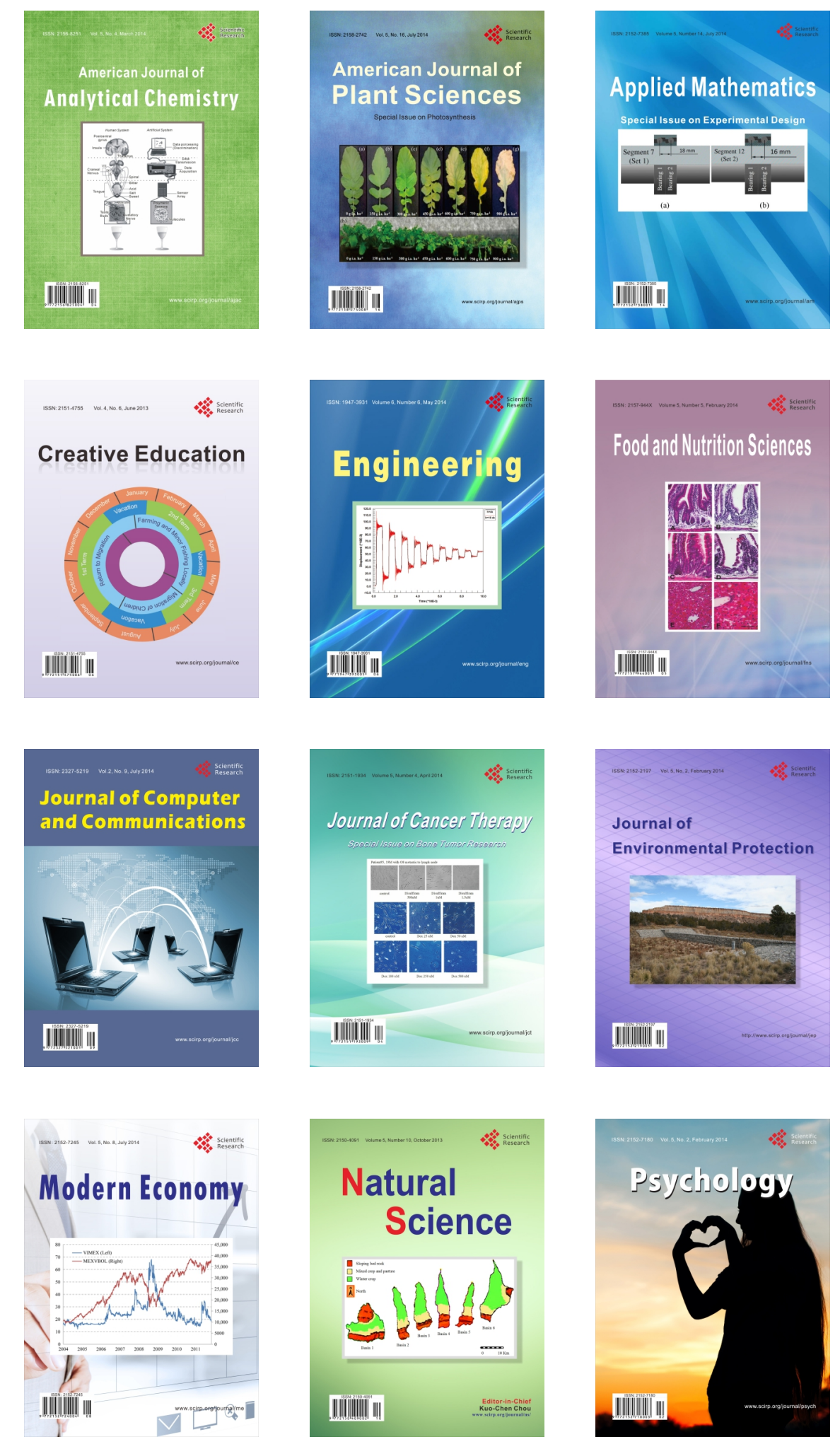